\title{
MISHCHENKO AND QUILLEN FORMULAS FOR ORIENTED COHOMOLOGY PRETHEORIES
}

\author{
E. SHINDER
}

\begin{abstract}
Two formulas mentioned in the title are generalized to arbitrary oriented cohomology pretheories on algebraic varieties.
\end{abstract}

\section{INTRODUCTION}

We generalize some well-known formulas and relations for complex cobordism to oriented cohomology pretheories on algebraic varieties. The definition and properties of oriented pretheories were given in $\mathrm{Pa}$.

Given an oriented cohomology pretheory, Mischenko's theorem expresses the coefficients of the related invariant differential form in terms of classes of projective spaces. For the complex cobordism theory, this theorem was stated and proved in Mishchenko's Appendix to $\mathrm{N}$. In the first part of the present paper, we generalize this theorem to an arbitrary oriented cohomology pretheory (see Theorem 1). The proof is based on the complex cobordism theorem mentioned above. For the first time, Mishchenko's theorem was proved in this generality by I. A. Panin in a slightly different way; we present it here for completeness.

Quillen's formula expresses the action of the direct image of a projection for projectivized bundles in terms of the formal group law and the invariant differential form that are related to the cohomology theory in question. This formula was stated without proof for the complex cobordism theory in [Qu1, p. 1294]. In the second part of the present paper, we generalize Quillen's formula to the case of an arbitrary theory. The proof is based on the Mishchenko theorem treated in the first part of the paper, rather than on the formula in Qu1. In this generality, Quillen's formula is a new result (see Theorem 3). The proofs of the two main theorems are based on Theorem 2 describing the direct image of an embedding of projectivized bundles.

The author thanks I. A. Panin and K. I. Pimenov for extremely helpful discussions.

In this paper, we use the following notation:

- $X$ is a smooth algebraic variety over an algebraically closed field $k$;

- $p t=\operatorname{Spec}(k)$;

$\mathbf{P}^{n}$ is the $n$-dimensional projective space over a field $k$;

- $\mathbf{1}_{X}^{n}$ is the trivial bundle of rank $n$ over $X$;

$E^{\vee}$ is the dual bundle for a bundle $E$;

$\mathbf{P}(E)$ is the projectivization of a bundle $E$;

$\mathcal{O}_{E}(-1)$ and $\mathcal{O}_{E}(1)$ are the tautological and the canonical bundle over $\mathbf{P}(E)$, respectively;

2000 Mathematics Subject Classification. Primary 14F43.

Key words and phrases. Complex cobordism theory, oriented cohomology pretheory, Quillen's formula.

(C)2007 American Mathematical Society 
- $A$ is an oriented cohomology pretheory $\mathrm{Pa}$;

$M U$ is the complex cobordism theory;

- $\mathcal{L}(D)$ is the line bundle corresponding to a divisor $D \subset X$ [Har];

- $c_{0}^{A}(E), c_{1}^{A}(E), \ldots, c_{n}^{A}(E)$ are the Chern classes of a bundle $E$ of rank $n$; $c_{\max }^{A}(E)=c_{n}^{A}(E)$ is the highest Chern class; $c_{t}^{A}(E)=c_{0}^{A}(E)+c_{1}^{A}(E) t+\cdots+c_{n}^{A}(E) t^{n}$ is the Chern polynomial; $\xi_{1}, \xi_{2}, \ldots, \xi_{n}$ are the Chern roots, i.e., the formal variables for which $\left(1+\xi_{1} t\right)(1+$ $\left.\xi_{2} t\right) \cdots\left(1+\xi_{n} t\right)=c_{t}(E)$

- $F_{A}\left(t_{1}, t_{2}\right)$ is the formal group law related to a pretheory $A$; $\omega_{A}(t)$ is the invariant differential form for a formal group law $F_{A}\left(t_{1}, t_{2}\right)$;

- $\left[\mathbf{P}^{n}\right]_{A}=p_{A}(1) \in A(p t)$ for a projection $p: \mathbf{P}^{n} \rightarrow X$; $\left[\mathbf{C P}^{n}\right]=\left[\mathbf{P}^{n}\right]_{M U} \in M U(p t)$.

\section{§1. The generalized Mishchenko theorem}

The following theorem was proved in $[\mathrm{N}$.

Theorem (Mischenko).

$$
\omega_{M U}(t)=\sum_{n=0}^{\infty}\left[\mathbf{C P}^{n}\right] t^{n} d t
$$

We use this fact to obtain the next statement.

Theorem 1 (generalized Mischenko theorem). Let A be an oriented cohomology pretheory. Then

$$
\omega_{A}(t)=\sum_{n=0}^{\infty}\left[\mathbf{P}^{n}\right]_{A} t^{n} d t .
$$

It is well known that the formal group law $F_{M U}$ is the universal commutative onedimensional formal group law, and the ring $M U(p t)$ coincides with the Lazard ring; see Qu1. Consequently, for every oriented cohomology pretheory $A$, there exists a unique ring homomorphism

$$
l_{A}: M U(p t) \rightarrow A(p t)
$$

such that

$$
\left(l_{A}\right)_{*} F_{M U}\left(t_{1}, t_{2}\right)=F_{A}\left(t_{1}, t_{2}\right),
$$

or equivalently,

$$
l_{A}\left(a_{i j}^{M U}\right)=a_{i j}^{A},
$$

where the $a_{i j}^{A}$ are the coefficients of the formal group law $F_{A}\left(t_{1}, t_{2}\right)$,

$$
F_{A}\left(t_{1}, t_{2}\right)=t_{1}+t_{2}+\sum_{i, j \geq 1} a_{i j}^{A} t_{1}^{i} t_{2}^{j}
$$

It will be proved that

$$
\left(l_{A}\right)\left(\left[\mathbf{C P}^{n}\right]\right)=\left[\mathbf{P}^{n}\right]_{A}
$$

for all $n \geq 0$.

First, we prove two lemmas. 
Lemma 1.1. Let $\Delta: \mathbf{P}^{n} \rightarrow \mathbf{P}^{n} \times \mathbf{P}^{n}$ be the diagonal embedding, and let $p_{i}: \mathbf{P}^{n} \times \mathbf{P}^{n} \rightarrow$ $\mathbf{P}^{n}, i=1,2$, be projections. Put $\zeta_{i}=c_{1}\left(p_{i}^{*} \mathcal{O}(1)\right)$. Then, in the ring $A\left(\mathbf{P}^{n} \times \mathbf{P}^{n}\right)=$ $A(p t)\left[\zeta_{1}, \zeta_{2}\right] /\left(\zeta_{1}^{n+1}, \zeta_{2}^{n+1}\right)$, we have

$$
\Delta_{A}(1)=\zeta_{1}^{n}+\zeta_{2}^{n}+\sum_{i, j \geq 1} b_{i j}^{A} \zeta_{1}^{i} \zeta_{2}^{j}
$$

where the $b_{i j}^{A} \in A(p t)$ are polynomials in the variables $a_{i j}^{A}$ with integral coefficients independent of $A$.

Proof. In the ring $A\left(\mathbf{P}^{n} \times \mathbf{P}^{n}\right)$, we have the relation

$$
\Delta_{A}(1)=c_{n}^{A}\left(p_{1}^{*} \mathcal{O}(1) \otimes p_{2}^{*} Q\right),
$$

where $\mathcal{O}(1)$ is the canonical bundle over $\mathbf{P}^{n}$ and $Q$ is the canonical quotient bundle over $\mathbf{P}^{n}$ occurring in the exact sequence

$$
0 \rightarrow \mathcal{O}(-1) \rightarrow V^{n+1} \rightarrow Q \rightarrow 0
$$

$\left(V^{n+1}\right.$ is the trivial $(n+1)$-dimensional bundle over $\left.\mathbf{P}^{n}\right)$.

Formula (4) is a special case of Theorem 2 (see below) for $X=\mathbf{P}^{n}, E=\mathcal{O}(-1), F=$ $V^{n+1}$, and $M=Q$.

We express the Chern class $c_{n}^{A}\left(p_{1}^{*} \mathcal{O}(1) \otimes p_{2}^{*} Q\right)$ on the right-hand side of (4) in terms of $\zeta_{1}$ and $\zeta_{2}$. For this, we use the formula that expresses the highest Chern class of the tensor product of two bundles in terms of the Chern classes of the factors:

$$
c_{n}\left(p_{1}^{*} \mathcal{O}(1) \otimes p_{2}^{*} Q\right)=\prod_{j=1}^{n} F_{A}\left(\zeta_{1}, \xi_{j}\right),
$$

where the $\xi_{j}$ are the Chern roots of the bundle $p_{2}^{*} Q$, i.e., the formal variables for which

$$
c_{t}\left(p_{2}^{*} Q\right)=1+c_{1}\left(p_{2}^{*} Q\right) t+\cdots+c_{n}\left(p_{2}^{*} Q\right) t^{n}=\left(1+t \xi_{1}\right) \cdots\left(1+t \xi_{n}\right) .
$$

We rearrange $F_{A}\left(\zeta_{1}, \xi_{j}\right)$ in (6) to obtain

$$
\begin{aligned}
& c_{n}\left(p_{1}^{*} \mathcal{O}(1) \otimes p_{2}^{*} Q\right) \\
& \quad=\left(\zeta_{1}+\xi_{1}+\sum_{i, j \geq 1} a_{i j}^{A} \zeta_{1}^{i} \xi_{1}^{j}\right)\left(\zeta_{1}+\xi_{2}+\sum_{i, j \geq 1} a_{i j}^{A} \zeta_{1}^{i} \xi_{2}^{j}\right) \cdots\left(\zeta_{1}+\xi_{n}+\sum_{i, j \geq 1} a_{i j}^{A} \zeta_{1}^{i} \xi_{n}^{j}\right) .
\end{aligned}
$$

Removing parentheses, we arrive at the formula

$$
c_{n}\left(p_{1}^{*} \mathcal{O}(1) \otimes p_{2}^{*} Q\right)=\zeta_{1}^{n}+c_{n}\left(p_{2}^{*} Q\right)+\sum_{i, j \geq 1} b_{i j}^{A} \zeta_{1}^{i} c_{j}\left(p_{2}^{*} Q\right),
$$

where the $b_{i j}^{A}$ satisfy the requirements of the lemma.

To prove formula (3), we calculate $c_{j}\left(p_{2}^{*} Q\right)=p_{2}^{A} c_{j}(Q)$. Application of the Cartan formula to the exact sequence (5) yields

$$
c_{t}(\mathcal{O}(-1)) c_{t}(Q)=1 .
$$

Since $c_{t}(\mathcal{O}(-1))=1-\zeta t$, we have

$$
c_{t}(Q)=\frac{1}{1-\zeta t}=1+\zeta t+\cdots+\zeta^{n} t^{n} .
$$

In other words, $c_{j}(Q)=\zeta^{j}$. Substituting these expressions in (7), we obtain (3).

Lemma 1.2. For every $n>0$, the following expansion is valid in $A(p t)$ :

$$
\left[\mathbf{P}^{n}\right]_{A}=\sum_{j=0}^{n-1} \alpha_{j}^{A}\left[\mathbf{P}^{j}\right]_{A},
$$


where the $\alpha_{j}^{A} \in A(p t)$ are polynomials in the variables $a_{i j}^{A}$ with integral coefficients independent of $A$.

Proof. Consider the projection $p_{1}: \mathbf{P}^{n} \times \mathbf{P}^{n} \rightarrow \mathbf{P}^{n}$ and its direct image

$$
\left(p_{1}\right)_{A}: A\left(\mathbf{P}^{n} \times \mathbf{P}^{n}\right) \rightarrow A\left(\mathbf{P}^{n}\right) .
$$

We calculate the action of $\left(p_{1}\right)_{A}$ on the additive generator $\zeta_{1}^{i} \zeta_{2}^{j}$ of the $A(p t)$-module $A\left(\mathbf{P}^{n} \times \mathbf{P}^{n}\right)$. We have

$$
\left(p_{1}\right)_{A}\left(\zeta_{1}^{i} \zeta_{2}^{j}\right)=\left(p_{1}\right)_{A}\left(\left(p_{1}\right)^{A}\left(\zeta^{i}\right) \zeta_{2}^{j}\right)=\zeta^{i}\left(p_{1}\right)_{A}\left(\zeta_{2}^{j}\right)=\zeta^{i}\left[\mathbf{P}^{n-j}\right]_{A} .
$$

In particular,

$$
\left(p_{1}\right)_{A}\left(\zeta_{1}^{n}\right)=\zeta^{n}\left[\mathbf{P}^{n}\right]_{A}, \quad\left(p_{1}\right)_{A}\left(\zeta_{2}^{n}\right)=1
$$

Now, we apply the operator $\left(p_{1}\right)_{A}$ to both sides of formula (3). Using Lemma 1.1 and the relation $\left(p_{1}\right)_{A} \Delta_{A}=i d$, we obtain

$$
1=\zeta^{n}\left[\mathbf{P}^{n}\right]_{A}+1+\sum_{i, j \geq 1} b_{i j}^{A} \zeta^{i}\left[\mathbf{P}^{n-j}\right]_{A} .
$$

Since the elements $1, \zeta, \ldots, \zeta^{n}$ are linearly independent over $A(p t)$, the coefficient at $\zeta^{n}$ on the right-hand side must be equal to zero. Hence, we obtain the required expansion (8). The properties of the coefficients $\alpha_{i}^{A}$ follow from the corresponding properties of the $b_{i j}^{A}$. The lemma is proved.

Corollary. For the coefficients $\alpha_{j}^{A}$ occurring in Lemma 2, we have

$$
l_{A}\left(\alpha_{j}^{M U}\right)=\alpha_{j}^{A} .
$$

Proposition. $\left(l_{A}\right)\left(\left[\mathbf{C P}^{n}\right]\right)=\left[\mathbf{P}^{n}\right]_{A}$.

Proof. We use induction on $n$. If $n=0$, the statement is trivial because $\left[\mathbf{P}^{0}\right]_{A}=1$. For $n>0$, we employ Lemma 2 two times: for the theory $M U$ and for the theory $A$. We obtain

$$
l_{A}\left(\left[\mathbf{C P}^{n}\right]\right)=l_{A}\left(\sum_{j=0}^{n-1} \alpha_{j}^{M U}\left[\mathbf{C} \mathbf{P}^{j}\right]\right)=\sum_{j=0}^{n-1} \alpha_{j}^{A}\left[\mathbf{P}^{j}\right]_{A}=\left[\mathbf{P}^{n}\right]_{A} .
$$

The proposition is proved.

Proof of Theorem 1 . Since the homomorphism $l_{A}$ takes the coefficients of $F_{M U}$ to those of $F_{A}$, we see that this homomorphism takes the coefficients of $\omega_{M U}$ to those of $\omega_{A}$. Using (11) and the above proposition, we obtain (2). The theorem is proved.

\section{§2. Generalized Quillen formula}

Theorem 2. Let $X$ be a smooth variety, and let

$$
0 \rightarrow E \rightarrow F \rightarrow M \rightarrow 0
$$

be an exact sequence of vector bundles over $X$. Consider the commutative diagram

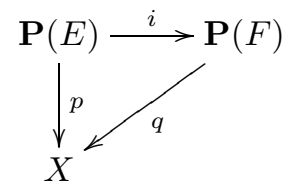

Then for the direct image $i_{A}$ of the morphism $i: \mathbf{P}(E) \rightarrow \mathbf{P}(F)$ we have

$$
\begin{aligned}
i_{A}(1) & =c_{\max }\left(\mathcal{O}_{F}(1) \otimes q^{*} M\right), \\
i_{A}\left(h\left(\zeta_{E}\right)\right) & =h\left(\zeta_{F}\right) c_{\max }\left(\mathcal{O}_{F}(1) \otimes q^{*} M\right),
\end{aligned}
$$


where $h(t)$ is an arbitrary polynomial with coefficients in $A(X), \zeta_{E}=c_{1}\left(\mathcal{O}_{E}(1)\right) \in$ $A(\mathbf{P}(E))$, and $\zeta_{F}=c_{1}\left(\mathcal{O}_{F}(1)\right) \in A(\mathbf{P}(F))$.

Proof. First, we note that relation (11) follows from (10) and the projection formula. To prove formula (10), we apply the splitting principle, by which we may assume that the sequence (9) is split, $F=M \oplus E$, and that $M=\bigoplus_{i=1}^{m} L_{i}$, where the $L_{i}, i=0, \ldots, m$, are line bundles. We use induction on $m$.

Base of induction: $m=1$. In this case, $i: P(E) \hookrightarrow P(F)$ is an embedding of a divisor, so that $i_{A}(1)=c(\mathcal{L})$, where $\mathcal{L}=L(\mathbf{P}(E))$. We prove that $\mathcal{L}=\mathcal{O}(1) \otimes q^{*} M$. First, we consider the case where $M=\mathbf{1}$. Then $F=\mathbf{1} \oplus E$ and $\mathcal{L}=\mathcal{O}_{1 \oplus E}(1)$, because the latter has a natural section $t$ (the first coordinate in $1 \oplus E$ ) whose zeros coincide with $\mathbf{P}(E)$. For an arbitrary $M$, we have

$$
\begin{aligned}
\mathbf{P}(M \oplus E) & =\mathbf{P}\left((M \oplus E) \otimes M^{\vee}\right)=\mathbf{P}\left(\mathbf{1} \oplus\left(E \otimes M^{\vee}\right)\right), \\
\mathcal{L} & =\mathcal{O}_{\mathbf{1} \oplus\left(E \otimes M^{\vee}\right)}(1)=\mathcal{O}_{M \oplus E}(1) \otimes q^{*} M .
\end{aligned}
$$

Induction step. Suppose $M_{k}=\bigoplus_{i=1}^{k} L_{i}$ and $F_{k}=E \oplus M_{k}$. We have the commutative diagram

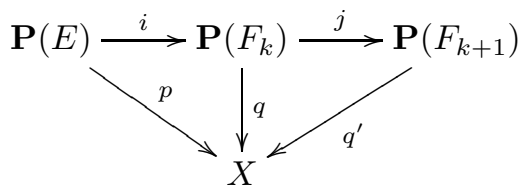

Let $i^{\prime}$ be the composition of $i$ and $j$. We must prove that

$$
i_{A}^{\prime}(1)=c_{k+1}\left(\mathcal{O}_{F_{k+1}}(1) \otimes q^{\prime *} M_{k+1}\right) .
$$

The exact sequence

$$
0 \rightarrow E \rightarrow F_{k} \rightarrow M_{k} \rightarrow 0
$$

and the inductive hypothesis imply that $i_{A}(1)=c_{k}\left(\mathcal{O}_{F_{k}}(1) \otimes q^{*} M_{k}\right)$, and the sequence

$$
0 \rightarrow F_{k} \rightarrow F_{k+1} \rightarrow L_{k} \rightarrow 0
$$

yields $j_{A}(1)=c_{1}\left(\mathcal{O}_{F_{k+1}}(1) \otimes q^{\prime *} L_{k}\right)$. Now, we calculate $i_{A}^{\prime}(1)$ :

$$
\begin{aligned}
i_{A}^{\prime}(1) & =j_{A}\left(i_{A}(1)\right)=j_{A}\left(c_{k}\left(\mathcal{O}_{F_{k}}(1) \otimes q^{*} M_{k}\right)\right) \\
& =j_{A}\left(j^{A}\left(c_{k}\left(\mathcal{O}_{F_{k+1}}(1) \otimes q^{\prime *} M_{k}\right)\right)\right)=j_{A}(1) c_{k}\left(\mathcal{O}_{F_{k+1}}(1) \otimes q^{\prime *} M_{k}\right) \\
& =c_{1}\left(\mathcal{O}_{F_{k+1}}(1) \otimes q^{\prime *} L_{k+1}\right) c_{k}\left(\mathcal{O}_{F_{k+1}}(1) \otimes q^{\prime *} M_{k}\right) \\
& =c_{k+1}\left(\mathcal{O}_{F_{k+1}}(1) \otimes q^{\prime *} M_{k+1}\right) .
\end{aligned}
$$

The theorem is proved.

Notation. For every vector bundle $E$ of rank $n$ over a smooth variety $X$, we consider the formal power series $f_{E}(t) \in A(X)[[t]]$ defined by the following formula:

$$
f_{E}(t)=\prod_{i=1}^{n}\left(t-{ }_{F} \lambda_{i}\right)
$$

where the $\lambda_{i}, i=1, \ldots, n$, are the Chern roots of the bundle $E$.

For example, we have $f_{E}(t)=t^{n}$ for $E=\mathbf{1}^{n}$.

Lemma 2.1. Consider the projection $\pi: X \times \mathbf{P}^{\infty} \rightarrow X$ and identify $A\left(X \times \mathbf{P}^{\infty}\right)$ with $A(X)[[t]], t=c_{1}(\mathcal{O}(1))$. Then in the ring $A\left(X \times \mathbf{P}^{\infty}\right)$ we have

$$
c_{n}\left(\mathcal{O}(1) \otimes \pi^{*} E\right)=f_{E^{\vee}}(t) .
$$


Proof. By the splitting principle, we may assume that

$$
E=\bigoplus_{i=1}^{n} L_{i}
$$

Then

$$
\begin{aligned}
c_{n}\left(\mathcal{O}(1) \otimes \pi^{*} E\right) & =c_{n}\left(\mathcal{O}(1) \otimes \pi^{*} \bigoplus_{i=1}^{n} L_{i}\right)=c_{n}\left(\bigoplus_{i=1}^{n}\left(\mathcal{O}(1) \otimes \pi^{*} L_{i}\right)\right) \\
& =\prod_{i=1}^{n} c_{1}\left(\mathcal{O}(1) \otimes \pi^{*} L_{i}\right)=\prod_{i=1}^{n} F_{A}\left(t, \lambda_{i}\right)=f_{E^{\vee}}(t) .
\end{aligned}
$$

Lemma 2.2. Let

$$
0 \rightarrow E^{\prime} \rightarrow E \rightarrow E^{\prime \prime} \rightarrow 0
$$

be an exact sequence of bundles over $X$. Then

$$
f_{E}(t)=f_{E^{\prime}}(t) f_{E^{\prime \prime}}(t) .
$$

Proof. It suffices to apply the Cartan formula to the Chern class of Lemma 2.1.

Lemma 2.3. The power series $f_{E}(t)$ is invertible in $A(X)((t))$.

Proof. By the splitting principle and the definition of $f_{E}(t)$, it suffices to prove the statement for a line bundle. In this case, the element $f_{E}(t)=t-{ }_{F} \lambda=F(t, I \lambda)=$ $t-\lambda+\sum_{i, j \geq 1} a_{i j} t^{i} \lambda^{j}=-\lambda+t(1+\nu+t(\cdots))$ is invertible, being the sum of a nilpotent and an invertible element.

Theorem 3 (Quillen's formula). Let $E$ be a bundle of rank $n$ over a smooth variety $X$. Let $\mathbf{P}(E)$ be its projectivization and $p: \mathbf{P}(E) \rightarrow X$ the projection. We denote by $\omega(t)$ the invariant differential form corresponding to $F_{A}\left(t_{1}, t_{2}\right)$. Then for the direct image $p_{A}$ we have

$$
p_{A}\left(\zeta_{E}^{k}\right)=\operatorname{Res}_{t=0} \frac{t^{k} \omega(t)}{f_{E^{\vee}}(t)},
$$

where $\zeta_{E}=c_{1}\left(\mathcal{O}_{E}(1)\right) \in A(\mathbf{P}(E))$.

Lemma 2.4. Formula (12) is valid for a trivial bundle $E$.

Proof. It is well known that the direct image of the projection $p: X \times \mathbf{P}^{n-1} \rightarrow p t$ acts by the formula

$$
p_{A}\left(\zeta^{k}\right)=\left[\mathbf{P}^{n-k-1}\right] .
$$

Rearranging the right-hand side of the above relation, we obtain

$$
\operatorname{Res}_{t=0} \frac{t^{k} \omega(t)}{f_{E^{\vee}}(t)}=\operatorname{Res}_{t=0} \frac{t^{k} \omega(t)}{t^{n}}=\operatorname{Res}_{t=0}\left(t^{k-n} \omega(t)\right) .
$$

Theorem 1 states that

$$
\omega_{A}(t)=\left(\left[\mathbf{P}^{0}\right]+\left[\mathbf{P}^{1}\right] t+\cdots+\left[\mathbf{P}^{n}\right] t^{n}+\cdots\right) d t .
$$

Therefore, the required residue is equal to $\left[\mathbf{P}^{n-k-1}\right]$, and the right-hand side in (12) coincides with the left-hand side, which proves the lemma.

Proof of Theorem 3. Using the Jouanolou trick [P1], we assume that $X$ is affine. On an affine variety, we have a natural equivalence between the category of vector bundles and the category of projective modules. Since a projective module is a direct summand of a free module, we obtain the following exact sequence of vector bundles over $X$ :

$$
0 \rightarrow E \rightarrow \mathbf{1}^{N} \rightarrow M \rightarrow 0
$$


Consider the commutative diagram

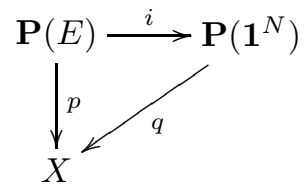

The direct image $p_{A}$ of $p$ can be calculated as the composition of the mappings $i_{A}$ and $q_{A}$. By Theorem 2,

$$
i_{A}\left(\zeta_{E}^{k}\right)=\zeta_{1^{N}}^{k} c_{m}\left(\mathcal{O}_{1^{N}}(1) \otimes q^{*} M\right)
$$

Arguing as in the proof of Lemma 2.1, it is easy to check that

$$
c_{m}\left(\mathcal{O}_{1^{N}}(1) \otimes q^{*} M\right)=\left.f_{M^{\vee}}(t)\right|_{t=\zeta} .
$$

Lemmas 2.2 and 2.3 allow us to find $f_{M \vee}(t)$ :

$$
f_{M^{\vee}}(t)=\frac{t^{N}}{f_{E^{\vee}}(t)} .
$$

As a result, we see that $i_{A}\left(\zeta_{E}^{k}\right)=\left.h(t)\right|_{t=\zeta_{1 N}}$ with $h(t)=\frac{t^{N+k}}{f_{E^{V}}(t)} \in A(X)[[t]]$. Substituting this expression in the formula for $q_{A}$ (see Lemma 2.4), we obtain

$$
p_{A}\left(\zeta_{E}^{k}\right)=q_{A}\left(i_{A}\left(\zeta_{E}^{k}\right)\right)=q_{A}\left(h\left(\zeta_{\mathbf{1}^{N}}\right)\right)=\operatorname{Res}_{t=0} \frac{h(t) \omega(t)}{t^{N}} .
$$

After simplification, we arrive at the formula

$$
p_{A}\left(\zeta_{E}^{k}\right)=\operatorname{Res}_{t=0} \frac{t^{k} \omega(t)}{f_{E^{\vee}}(t)} .
$$

The theorem is proved.

Corollary. Under the assumptions and in the notation of Theorem 2, we have

$$
p_{A}(h(\zeta))=\operatorname{Res}_{t=0} \frac{h(t) \omega(t)}{f_{E^{\vee}}(t)},
$$

where $h(t)$ is an arbitrary polynomial with coefficients in $A(X)$.

Proof. Formula (13) follows from (12) and the theorem on projectivized bundles.

\section{REFERENCES}

[Har] R. Hartshorne, Algebraic geometry, Grad. Texts in Math., No. 52, Springer-Verlag, New YorkHeidelberg, 1977. MR 0463157 (57:3116)

[P1] I. Panin, Push-forwards in oriented cohomology theories of algebraic varieties. II, http://www. math.uiuc.edu/K-theory/0619/2003.

[PS] I. Panin and A. Smirnov, Push-forwards in oriented cohomology theories of algebraic varieties, http://www.math.uiuc.edu/K-theory/0459/2000.

[Pa] I. Panin, Riemann-Roch theorems for oriented cohomology, Axiomatic, Enriched, and Motivic Homotopy Theory, NATO Sci. Ser. II Math. Phys. Chem., vol. 131, Kluwer Acad. Publ., Dordrecht, 2004, pp. 261-333. MR2061857 (2005g:14025) 
[Qu1] D. Quillen, On the formal group laws of unoriented and complex cobordism theory, Bull. Amer. Math. Soc. 75 (1969), 1293-1298. MR0253350 (40:6565)

[N] S. P. Novikov, Methods of algebraic topology from the point of view of cobordism theory, Izv. Akad. Nauk SSSR Ser. Mat. 31 (1967), no. 4, 855-951; English transl. in USSR-Izv. 1 (1967). MR0221509 (36:4561)

Department of Mathematics and Mechanics, St. Petersburg State University, UniverSitetskiI Prospect 28, Stary ̌ Peterhof, St. Petersburg 198904, Russia

E-mail address: shinder@list.ru

Received 22/FEB/2006

Translated by B. M. BEKKER 